\title{
Dust and metal column densities in GRB host galaxies
}

\author{
P. Schady ${ }^{1}, *$ T. Dwelly ${ }^{1}$ M. J. Page ${ }^{2}$ J. Greiner ${ }^{1}$ T. Krühler ${ }^{3}$ \\ S. Savaglio ${ }^{1}$ S. Oates ${ }^{2}$ A. Rau ${ }^{1}$ and the GROND and UVOT teams \\ ${ }^{1}$ Max-Planck-Institut für Extraterrestrische Physik, Giessenbackstraße 1, 85748 Garching, \\ Germany \\ *email: pschady@mpe.mpg.de \\ ${ }^{2}$ The UCL Mullard Space Science Laboratory, Holmbury St Mary, Surrey, RH5 6NT, UK \\ ${ }^{3}$ Dark Cosmology Centre, Niels Bohr Institute, University of Copenhagen, Juliane Maries Vej \\ 30, 2100 Copenhagen, Denmark
}

\begin{abstract}
The immensely bright and intrinsically simple afterglow spectra of gamma-ray bursts (GRBs) have proven to be highly effective probes of the interstellar dust and gas in distant, star-forming galaxies. Despite significant progress, many aspects of the host galaxy attenuating material are still poorly understood. There is considerable discrepancy between the amount of X-ray and optical afterglow absorption, with the former typically an order of magnitude higher than what would be expected from the optical line absorption of neutral element species. Similar inconsistencies exist between the abundance of interstellar dust derived from spectroscopic and photometric data, and the relation between the line-of-sight and integrated host galaxy interstellar medium (ISM) remains unclear. In these proceedings we present our analysis on both spectroscopic and photometric multi-wavelength GRB afterglow data, and summarise some of the more recent results on the attenuation properties of the ISM within GRB host galaxies.
\end{abstract}

Keywords. gamma rays: bursts, galaxies: ISM, ISM: dust, extinction, ISM: abundances

\section{Introduction}

A causal connection between long gamma-ray bursts (GRBs) and massive star formation is now well established, and their immensely bright afterglows therefore pinpoint regions of distant star formation independent of galaxy luminosity. Moreover, due to their featureless, broadband afterglow, the imprint from attenuating material within the host galaxy can be studied in detail, providing a truly unique view of the interstellar medium (ISM) within distant star forming galaxies. Their incredibly bright and multi-wavelength afterglow not only illuminates the gas and dust within the star forming regions of the host galaxy, but also that of the interstellar material in the disk and halo of the galaxy, and intervening intergalactic medium along the GRB line-of-sight.

In these proceedings we summarise our work in the context of others on the various components of attenuating gas, metals and dust within the host galaxies of GRBs. Our analysis makes use of X-ray through to near-infrared (nIR) spectroscopic and photometric data, comprising one of the most complete studies on the attenuation properties of the ISM within GRB host galaxies. In particular, we shall address the nature of the excess X-ray absorption and discuss the location of this "missing" absorbing gas, as well as discuss the observed variation in the dust extinction properties of GRB host galaxies.

These proceedings are divided into two main sections, where in section 2 we focus on afterglow absorption from intervening gas and metals, and in section 3 we discuss properties of dust extinction along the GRB line-of-sight, and its relation with the global host galaxy characteristics. A summary and future prospects is provided in section 4 . 


\section{Afterglow absorption from intervening gas and metals}

From the increasing sample of GRB spectroscopic observations that cover the neutral hydrogen Lyman- $\alpha$ absorption feature at ultraviolet (UV) wavelengths (rest-frame $1215 \AA$ ), it is becoming clear that GRB host galaxies have high column densities of cold neutral gas $\left(T \leqslant 10^{3} \mathrm{~K}\right)$. Any corresponding ionised hydrogen would not be detected, and neutral hydrogen measurements thus require an ionisation correction to determine the total column density of atomic hydrogen, $N_{\mathrm{H}}$. A large fraction of GRB hosts have such large $N_{\mathrm{H}}$ values, however, that the ionisation correction is negligible and the neutral absorbing gas component is known as a damped-Lyman- $\alpha$ (DLA) system $\left(\log \left(\mathrm{N}_{\mathrm{HI}} / \mathrm{cm}^{-2}\right)>20.3\right)$. The survival of certain species, such as $\mathrm{Mg}$ I, and time varying Fe II and Ni II finestructure lines, place this neutral gas component at a few hundred parsecs from the GRB (e.g. Vreeswijk et al. 2007 ), within the ISM of the host galaxy. The neutral ISM is also traced by low ionisation species detected in the UV, whereas highly-ionised species (e.g. O IV, C IV, Si IV and N V) possibly probe the hot gas (T $\sim 10^{4} \mathrm{~K}$ ) within the circumburst environment of the GRB (Prochaska et al. 2008), as well as a contribution from gas in the rest of the galaxy (Fox et al. 2008).

In contrast to the specific regions of gas that can be identified from UV spectra, Xray spectroscopic observations provide measurements of the total column density of gas along the line-of-sight, probing both the cold neutral gas as well as the warm ionised regions. Soft X-rays with energies $<0.8 \mathrm{keV}$ are absorbed by medium-weight metals along the line-of-sight, which is predominantly in the form of oxygen and to a lesser extent carbon and nitrogen. Furthermore, the cross-section of oxygen remains relatively unchanged regardless of ionisation state (Verner \& Yakovlev 1995), making it a good proxy for the host galaxy total oxygen column density. In addition to this, soft X-rays can also be absorbed by metals locked in dust grains, although optical afterglow dust extinction measurements indicate that most GRB lines-of-sight are relatively dust-poor.

In comparing X-ray and optical afterglow measurements, Watson et al. 2007 noted considerable discrepancy between the two. They found the equivalent neutral hydrogen column density derived from soft X-ray absorption (assuming solar abundances), $N_{\mathrm{H}, \mathrm{X}}$, to be typically an order of magnitude higher than the optically derived $N_{\mathrm{H}}$. This led the authors to conclude that the former probed a significant column of ionised gas that is transparent to UV photons.

In order to explore the properties of this additional absorbing component, in Schady et al. (2011) we combined the results from the analysis of optical and X-ray spectra, and also of broadband afterglow SEDs, with the aim of providing greater insight on the ionisation state of the host galaxy medium along the line-of-sight to GRBs. Instead of neutral hydrogen, we used absorption lines of singly-ionised metals to trace the neutral gas within GRB host galaxies that could then be compared directly with the soft X-ray absorption, which measures primarily the oxygen column density (neutral and ionised), $N_{\mathrm{O}, \mathrm{X}}$. This removes the need for hydrogen column density and host galaxy metallicity measurements, which are frequently uncertain or unavailable, thus increasing our sample size. We took a sample of $26 \mathrm{GRBs}$ with detected optical spectroscopic absorption lines in at least one of Zn II, Si II, S II or Fe II with which to probe the host galaxy neutral gas, as well as with detected soft X-ray absorption or upper limit.

\subsection{Total versus neutral gas}

To trace the neutral gas within a GRB host galaxy, we preferentially used the ion Zn II or S II, which if not available was substituted by Si II or otherwise Fe II. This is due to the inherent uncertainties present in the dust depletion of the refractory elements Si II, and 

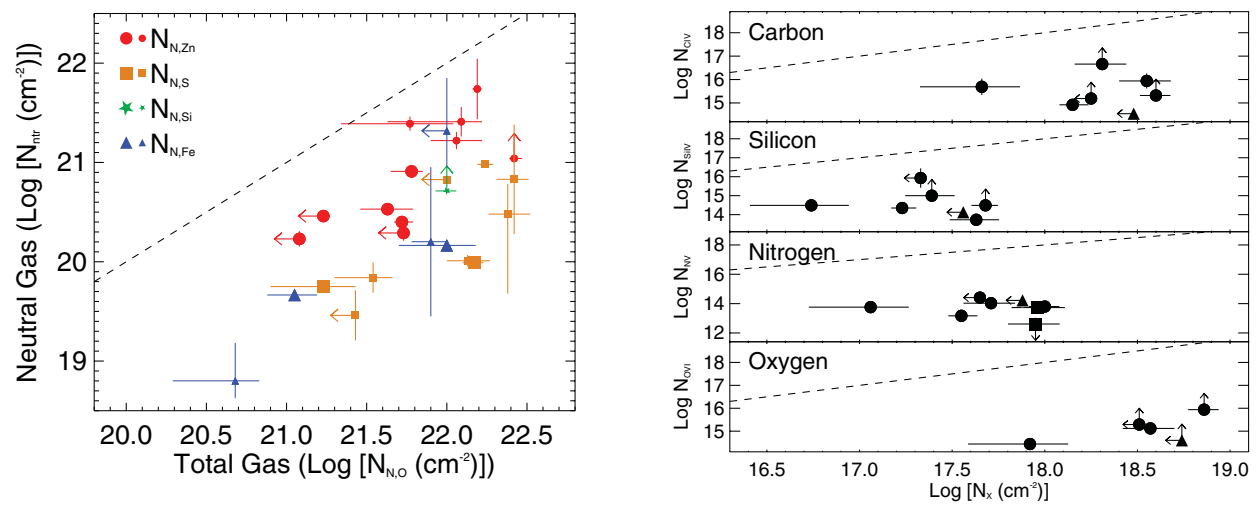

Figure 1. Left: Host galaxy neutral gas column density, $N_{\mathrm{ntr}}$, against host galaxy total gas column density, $N_{\mathrm{N}, \mathrm{O}}$, along the line-of-sight to a sample of $26 \mathrm{GRBs}$. For each GRB, $N_{\mathrm{ntr}}$ is derived from either the column density of Zn II (red circles), S II (orange squares), Si II (green stars) or Fe II (blue triangles), where a correction for dust depletion is applied to $N_{\mathrm{N}, \mathrm{Si}}$ and $N_{\mathrm{N}}$, Fe. Smaller data points correspond to those taken from lowor mid-resolution spectra, and larger data points are taken from high-resolution spectra $(\mathrm{R}>10,000)$. The dashed line corresponds to where $N_{\text {ntr }}$ is equal to $N_{\mathrm{N}, \mathrm{O}}$. Right: Logarithmic host galaxy column density of the highly ionised atoms C IV, Si IV, N V and O VI against the total logarithmic column densities of C, Si, N and O in the top, second, third and bottom panels, respectively. The total column densities are derived from X-ray observations, denoted by $N_{X}$. The C IV, Si IV, N V and O vi measurements plotted as circles are all taken from Fox et al. (2008), the N v data plotted as squares are from Prochaska et al. (2008), and data taken from D'Elia et al. (2010) are plotted as triangles. In all four panels, the dashed line corresponds to where the normalised soft X-ray column density is equal to the column density of the corresponding highly ionised atom.

especially Fe II. When column densities for both Zn II and S II were available, the one with the smaller error bars was used. When only Si II or Fe II absorption measurements were available, a dust correction was applied to the measured column density. We then corrected for cosmic abundance variances by normalising all our measurements to each other using the abundances from Asplund et al. (2009). We denote the normalised column densities of oxygen, zinc, silicon, sulphur and iron by $N_{N, O}, N_{N, Z n}, N_{N, S i}, N_{N, S}$ and $N_{N, F e}$, respectively.

In the left panel of Fig. 1 we show the neutral gas column density, $N_{\text {ntr }}$, represented by either $N_{\mathrm{N}, \mathrm{Zn}}$ (red circles), $N_{\mathrm{N}, \mathrm{S}}$ (orange squares), $N_{\mathrm{N}, \mathrm{Si}}$ (green stars) or $N_{\mathrm{N}, \mathrm{Fe}}$ (blue triangles), against $N_{\mathrm{N}, \mathrm{O}}$, which represents the total column of gas (neutral and partially ionised), where both $N_{\mathrm{N}, \mathrm{Si}}$ and $N_{\mathrm{N}, \mathrm{Fe}}$ have been corrected for dust depletion. The dashed line in Fig. 1 (left panel) corresponds to where the column density of neutral gas is equal to the total column density of gas as probed by our X-ray absorption measurements. From this figure it is clear that for all GRBs, $N_{\mathrm{N}, \mathrm{O}}$ is larger than the column density of neutral gas, $N_{\text {ntr }}$, by around an order of magnitude, implying that over $90 \%$ of the gas along the line-of-sight is ionised.

There is an indication that the difference between the soft X-ray and neutral metal absorption increases for smaller column densities. This would suggest that the fraction of ionised gas is not dependent on the global galaxy properties, but on local conditions, such as would be the case if the GRB itself were the dominant source of ionising photons. Nevertheless, this trend is not significant, and we can thus only speculative at this stage.

\subsection{Highly ionised gas}

To explore further the ionisation state of the X-ray absorbing gas, we looked into the contribution from highly ionised gas, such as C IV, Si IV, N V and O VI, that unlike lowionised gas, can survive within the circumburst environment of the GRB (Fox et al. 2008; 
Prochaska et al. 2008). We used the high-ionised gas absorption measurements for nine GRBs reported in Fox et al. (2008), Prochaska et al. (2008), and D'Elia et al. (2010). To compare the amount of soft X-ray absorbing gas with the fraction of highly ionised gas, we then normalised the soft X-ray absorption measurements to the cosmic abundance of the element in question being compared (i.e. either Si, C, N or O). Our results are shown in Fig. 1 (right panel), which compares the logarithmic column density of the highly ionised atoms $N_{C I V}, N_{S I V}, N_{N V}$ and $N_{O V I}$ against the soft X-ray absorption column densities normalised to $\mathrm{C}, \mathrm{Si}, \mathrm{N}$ and $\mathrm{O}$ cosmic abundances in the top, second, third and bottom panel, respectively. The normalised soft X-ray absorption column density is denoted by $N_{X}$ to indicate the column density of C, Si, N or O as determined from our soft X-ray absorption measurements. In all four panels the dashed line represents where the normalised soft X-ray column density measurements are equal to the column density of the highly ionised atom represented in each corresponding panel.

It is clear from this figure that all data points lie several orders of magnitude below the dashed lines, indicating that the highly ionised gas within GRB hosts only makes up a small fraction $(<0.01 \%)$ of the soft X-ray absorption. The left-hand panel in Fig. 1, on the other hand, indicates that $\sim 90 \%$ of the gas probed by the soft X-rays is ionised. If the large fraction of this gas is in a lower ionised state, then there should be a signature of this in the detection of strong absorption lines from intermediate ionisation lines with ionising potentials (IPs) between $\sim 20 \mathrm{eV}$ and $\sim 50 \mathrm{eV}$ (i.e. Si II and Si IV). This is, however, not the case. For example, Al III, which has an IP of $28 \mathrm{eV}$, is frequently observed to be weaker than Al II, which has an IP of $19 \mathrm{eV}$. This would, therefore, suggest that most of the ionised gas probed by the soft X-rays is in an ultra-ionised state, with IPs larger than $\sim 200 \mathrm{eV}$. The signature left by such an ultra-ionised gas would lie predominantly in the soft X-ray energy range, which is already heavily absorbed by oxygen and carbon (both neutral and ionised). Resolving the absorption from an ultra-ionised gas is, therefore, beyond the spectral capabilities of current fast-response X-ray telescopes and will require a future era of rapid-response, high-resolution X-ray telescopes.

Other possibilities that have been raised to explain the origins of this soft X-ray excess absorption have been an underestimate of the absorption from metals within the Milky Way, absorption from intervening systems (Campana et al. 2012), or from a diffuse and local 'warm-hot' intergalactic medium, or WHIM (Behar et al. 2011).

\section{Afterglow dust extinction}

Another source of attenuation is in the absorption and scattering of light, or extinction, caused by intervening dust. The amount of dust extinction along a single line-of-sight is, for the most part, an inverse function of wavelength, and this dependence on wavelength is known as the dust extinction curve. The extinction curve is a function of both the grain size distribution and composition of the extinguishing dust, and the effect that differences in environmental conditions have on the observed extinction curve can be seen within the local Universe, in the average extinction curve observed within the Milky Way, and the Large and Small Magellanic Clouds (LMC and SMC respectively) (see Fig. 2). The dominant differences between these extinction curves is in the prominence of an extinction feature centred at $\sim 2175 \AA$ (most pronounced along Milky Way sight lines, negligible within the SMC), and in the slope of the extinction curve at UV and optical wavelengths, which is flattest within the Milky Way, and gets progressively steeper from LMC to SMC sight lines.

The single or broken power-law GRB afterglow spectrum allows the broad, smooth shape cut-out by dust-extinction to be measured with relative ease, making GRBs ideal 


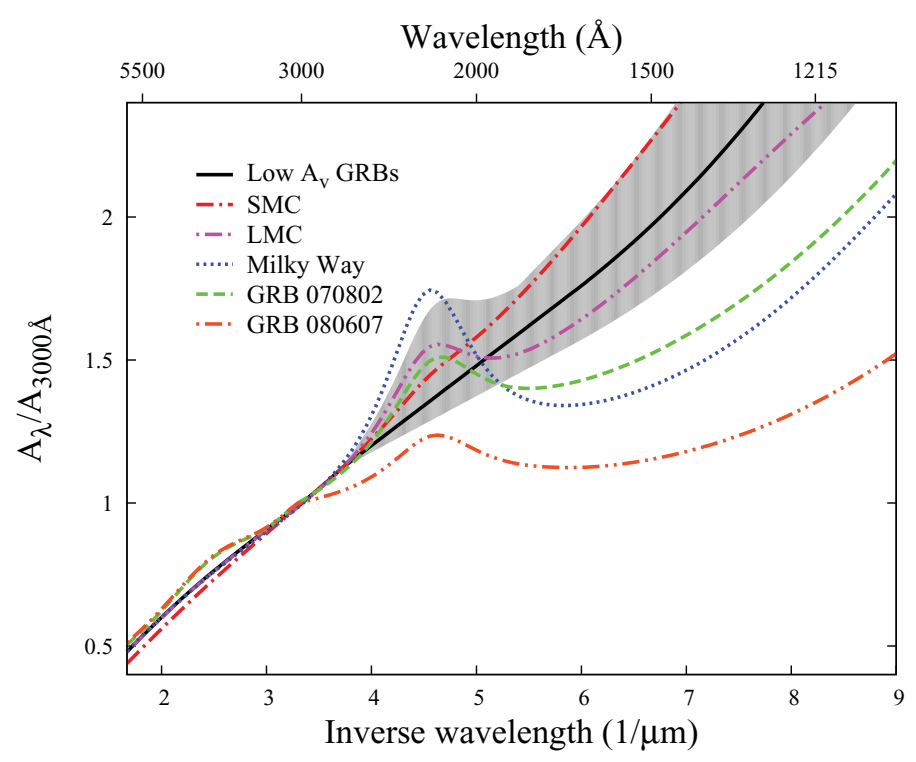

Figure 2. Best-fit mean GRB host extinction law as derived from simultaneous SED fits normalised at $A_{3000 \AA}$ (black) and corresponding 90\% confidence region (grey) (Schady et al. 2012). Also shown for comparison are the mean SMC (red, dot-dash), LMC (pink, dot-dot-dash) and Milky Way (blue, dotted) extinction laws (Pei 1992), as well as the best-fit extinction curves to the two heavily extinguished GRBs, GRB 070802 (green, dashed; Elíasdóttir et al. 2009) and GRB 080607 (orange, dot-dot-dashed; Perley et al. 2011).

probes to the extinction properties of dust beyond the local Universe. Typically it is found that for GRBs with well-observed afterglow spectral energy distributions (SEDs), the amount of host galaxy extinction is small (total $V$-band or visual extinction $\left\langle A_{\mathrm{V}}\right\rangle<0.3$ (e.g Kann et al. 2006, Schady et al. 2010, Schady et al. 2012), and has a nIR to UV wavelength dependence similar to that along SMC lines-of-sight. However, recent dedicated GRB rapid-response programmes with nIR wavelength coverage are now revealing a sample of GRBs $(\sim 10 \%)$ that have been significantly extinguished $\left(A_{V}>1\right)$ by host galaxy dust (Greiner et al. 2011), many of which show evidence for much flatter, Milky Way-like host extinction curves than observed in the hosts of only moderately extinguished GRBs. There are now four GRBs with spectroscopically confirmed detections of the $2175 \AA$ absorption bump at the redshift of the GRB host galaxy (Zafar et al. 2011), as well as several GRBs with a $2175 \AA$ extinction feature detected in the GRB SED. The causes for this range in the total host galaxy dust extinction, and in the shape of the extinction curves of GRB hosts are still poorly understood.

To investigate the origin of these observed variations further, in Schady et al.(2012) we used predominantly Swift data to quantify the range observed in GRB host extinction curves and investigate the possible environmental factors contributing to the measured variations. The results from this study were consistent with a dustier host galaxy having a flatter GRB host extinction curve, and more prominent $2175 \AA$ bump (see Fig. 2). Nevertheless, this analysis also emphasised the need for accurate, near-IR photometry to be able to increase the sample to include more dust-extinguished GRB afterglows, as well as to better study the relation between $A_{V}$ and the extinction curve properties.

\subsection{Heavily dust-extinguished GRB sightlines}

Around $40 \%$ of GRBs have their optical afterglow attenuated by either neutral gas within the intergalactic medium, or more commonly, from dust within the host galaxy (Perley 
et al. 2009, Greiner et al. 2011). For these so-called 'dark' GRBs, the arcsecond position provided by the optical afterglow is therefore not available, introducing a clear bias against follow-up observations of their host galaxies. The improved positional accuracy of GRBs provided by the rapid response of Swift and (semi-)robotic ground-based telecopes, in particular those equipped with nIR instruments, has significantly improved our capabilities over the last half decade to study highly dust-extinguished GRBs and their host galaxies. A leading instrument in acquiring broad-band observations that extend into the near-IR wavelength range has been the GRB optical and nIR detector (GROND), commissioned in July 2007. GROND is unique in providing simultaneous $g^{\prime} r^{\prime} i^{\prime} z^{\prime}$ and near-IR $J H K$ observations of the early-time afterglow, and has proved highly effective at detecting and measuring host galaxy dust extinction for a large sample of GRBs (Greiner et al. 2011), and increasing the host galaxy visual extinction distribution out to $A_{V}>1$. Other nIR instruments such as PAIRITEL have also contributed.

This reduction in the selection effects against dusty lines-of-sight has also seen an increase in the variety of GRB host galaxy properties. The majority of optically-selected host galaxies, making up the majority of the population, are young, star-forming, lowmass galaxies (Le Floc'h et al. 2003; Savaglio et al. 2009), with sub-solar metallicities. Recent follow-up observations of the host galaxies of heavily extinguished GRBs have nevertheless indicated the environmental conditions traced by GRBs to be much more diverse than indicated by previous, optically biased observations.

\subsubsection{Relation of extinction curves with $A_{V}$ and global galaxy properties}

There is empirical evidence that the prominence of the $2175 \AA$ extinction bump and the overall flatness of the extinction curve is related to the total visual extinction, $A_{V}$, along the line-of-sight. The few GRB host extinction curves with spectroscopically detected $2175 \AA$ extinction bump, also correspond to lines-of-sight with the largest visual extinction. There is some evidence that the host galaxies of highly dust-extinguished GRBs are typically more massive, luminous, and chemically evolved than the typical host galaxies of relatively unextinguished GRBs (e.g. Krühler et al. 2011). Nevertheless, despite such observed trends, the relation between the GRB line-of-sight, and the global host galaxy properties remains far from unclear.

One of the most dust-extinction GRBs (GRB 080607; Prochaska et al. 2009) also had one of the flattest extinction curves and most prominent $2175 \AA$ bumps detected along a GRB line-of-sight. Its host galaxy properties were also atypical when compared to optically-selected samples (Chen et al. 2011), with a stellar mass $M_{*} \sim 8 \times 10^{9} M_{\odot}$, almost an order of magnitude larger than the mean stellar mass of optically selected samples $\left(\left\langle M_{*}\right\rangle \sim 10^{9} M_{\odot}\right.$; Savaglio et al. 2009), as well as being highly reddened $(R-K>5$; Chen et al. 2011). This is also the only GRB to have a robust detection of molecular hydrogen absorption in its afterglow spectrum (Prochaska et al. 2009).

On the other hand, GRB 070306 and GRB 100621A were two other heavily extinguished GRBs $\left(A_{V} \sim 5.5\right.$ and $A_{V} \sim 3.8$ respectively), but both with very blue host galaxies, with $R-K$ colours comparable to the host galaxies of relatively unextinguished GRBs (Krühler et al. 2011), indicative of a very clumpy distribution of dust. Furthermore, although GRB 070306 had one of the largest stellar masses measured for a long GRB host galaxy $\left(2 \times 10^{10} M_{\odot}\right.$; Krühler et al. 2011), the host stellar mass for GRB 100621 $\left(10^{9} M_{\odot}\right)$ was comparable to that of optically bright afterglow host galaxies.

\subsubsection{Dust-to-metals ratio}

The visual extinction-to-metals column density ratio, $A_{V} / N_{\mathrm{H}, \mathrm{X}}$, within GRB host galaxies along the GRB line-of-sight has been investigated in a number of papers, and 
these ratios have been typically found to be much higher than the ones observed in the Local Group (e.g. Galama \& Wijers et al. 2001, Stratta et al. 2004, Starling et al. 2007, Schady et al. 2007,2010). The large majority of these studies have, nevertheless, been biased towards unextinguished lines-of-sight, and recent samples of GRBs with $A_{V}>4$ have shown metals-to-dust ratios significantly below what is typically measured for GRB afterglows, and more in line with measurements from the Local Group (Krühler et al. 2011). Krühler et al.(2011) also found a strong anti-correlation between the metals-todust ratio and $A_{V}$ along the GRB sight-line, which they speculate could be evidence of two physically independent absorbers: a dust-free, ionized plasma in the GRB circumburst environment, and another dusty and more distant, thus less ionised cloud.

\section{Summary and future prospects}

The move towards combining both imaging and spectroscopic data from across the spectrum, and ongoing programmes dedicated at pushing down observational biases in GRB afterglow and host galaxy samples are providing us which a much more rounded view on the local and global environments of GRBs, and on the effect that the GRB explosion has on the surrounding circumburst and interstellar material.

The large UV radiation field implied by a situation in which $\sim 90 \%$ of the gas within GRB host galaxies is in an ultra-ionised state has consequences for the effect of massive star formation on the surrounding environment. Direct detection of an ultra-ionised gas component would require early-time, high spectral-resolution X-ray afterglow data, which is currently unattainable. Nevertheless, a useful verification would be to model the UV radiation field and circum- and interstellar properties required to satisfy observations.

A significant contribution to the absorption of low-energy X-rays from a WHIM component, on the other hand, would have important implications to our understanding and investigation of 'the missing baryons problem'. The contribution to soft X-ray absorption from intervening gas external to the host galaxy could be further investigated through analysis on the dependence of the X-ray-to-optical afterglow absorption on redshift.

Finally, the tantalising evidence that GRBs may reside in more varied environmental conditions than previously speculated (i.e. with no metallicity cap), would suggest that GRBs make better tracers of the cosmic star formation rate density than had been thought, and certainly places GRBs as truly unique probes of the dust properties and ionisation state of the ISM within high- $z$, star-forming galaxies. How the attenuation along the GRB sight line relates to the global host galaxy properties is not yet clear, and this requires further investigation through dedicated, broadband (including nIR) afterglow follow-up campaigns to acquire a larger sample of unbiased, and thus more representative GRB afterglow and host galaxy observations.

\section{References}

Asplund, M., et al. 2009, AEA\&A, 47, 481

Behar, E., et al. 2011, ApJ, 734, 26

Campana, S., et al. 2012, MNRAS, 421, 169

Chen, H.-W., et al. 2011, ApJ, 727, L53

D'Elia, V., et al. 2010, $A \& A$ A, 523, 36

Elíasdóttir, Á., et al. 2009, ApJ, 697, 1725

Fox et al. 2008, A\& $A, 491,189$

Galama, T. J. \& Wijers, R. A. M. J. 2001, ApJ, 549, L209

Greiner, J., et al. 2011, A\& A, 526, A30

Kann, D. A., Klose, S., \& Zeh, A. 2006, ApJ, 641, 993 
Krühler, T., et al. 2011, A\& A, 534, 108

Pei, Y. C. 1992, ApJ, 395, 130

Perley, D. A., et al. 2009, AJ, 138, 1690

Perley, D. A., et al. 2011, AJ, 141, 36

Prochaska, J. X., et al. 2008, ApJ, 685, 344

Prochaska, J. X., et al. 2009, ApJ, 691, L27

Savaglio, S., Glazebrook, K., \& Le Borgne, D. 2009, ApJ, 691, 182

Schady, P., et al. 2007, MNRAS, 377, 273

Schady, P., et al. 2010, MNRAS, 401, 2773

Schady, P., et al. 2011, A\&A, 525, 113

Schady, P., et al. 2012, A\&A, 537, 15

Starling, R. L. C., et al. 2007, ApJ, 661, 787

Stratta, G., Fiore, F., Antonelli, L. A., Piro, L., \& De Pasquale, M. 2004, ApJ, 608, 846

Vreeswijk et al. 2007, A\&A, 468, 83

Verner, D. A., \& Yakovlev, D. G. 1995, A\&AS, 109, 124

Watson, D., et al. 2006, ApJ, 652, 1011

Watson, D., et al. 2007, ApJ, 660, 101

Zafar, D., et al. 2011, A\&BA, 532, 143

\section{Discussion}

DAVID BurRows: X-ray absorption traces the total column density of metals, not just the gas phase. Also, it is difficult to get substantial X-ray absorption from highly (ultra-) ionised gas, because the temperature of that gas is very high, and the density is very low unless it is transient.

SCHADY: I agree on both those points. On the first point, I do not, however, believe that the contribution to the X-ray absorption from metals locked up in dust is large. The particular sample of GRBs that I used in my investigation into the soft X-ray excess had, by selection, high signal-to-noise optical afterglow spectra, and thus little dust extinction. As to the latter point, I would certainly argue that if the "missing gas" is indeed in a ultra-ionised state, then it must be the GRB itself that produced the high UV-radiation field required to form such a gas.

RhaAna Starling: Do your results suggest a solar abundance pattern is correct for GRB hosts?

SCHADY: There is currently no evidence to believe otherwise. However, a different host galaxy solar abundance pattern to solar, such as an over-abundance of $\alpha$-elements, cannot account for the large soft X-ray absorption excess observed.

DANiel Perley: You mentioned that one explanation for the discrepancy between Xray inferred gas abundances and abundances inferred from optical spectroscopy could be the presence of gas in a very highly ionised state. Of course, the GRB itself is a prolific source of ionising radiation. Can you address the possibility that ionisation from the GRB could be the explanation for this discrepancy?

SCHADY: As already highlighted by David, such a highly ionised gas cannot be in equilibrium, and must thus be in a transient state. In this case, ionisation by the GRB itself could produce the short-lived, highly ionised gas. Furthermore, the most plausible location for this highly ionised plasma would be within a dense region very near-in to the GRB, which again, would favour the GRB as the source of the ionising photons. Further modelling would, nevertheless, be necessary to test the validity of such a scenario. 